\title{
MEASURABLE PARAMETRIZATIONS AND SELECTIONS
}

BY

\author{
DOUGLAS CENZER AND R. DANIEL MAULDIN
}

\begin{abstract}
Let $W$ be a Borel subset of $I \times I$ (where $I=[0,1]$ such that, for each $x, W_{x}=\{y:(x, y) \in W\}$ is uncountable. It is shown that there is a map, $g$, of $I \times I$ onto $W$ such that (1) for each $x, g(x, \cdot)$ is a Borel isomorphism of $I$ onto $W_{x}$ and (2) both $g$ and $g^{-1}$ are $S(I \times I)$-measurable maps. Here, if $X$ is a topological space, $S(X)$ is the smallest family containing the open subsets of $X$ which is closed under operation (A) and complementation. Notice that $S(X)$ is a subfamily of the universally or absolutely measurable subsets of $X$. This result answers a problem of $A$. $H$. Stone.

This result improves a theorem of Wesley and as a corollary a selection theorem is obtained which extends the measurable selection theorem of von Neumann.

We also show an analogous result holds if $W$ is only assumed to be analytic.
\end{abstract}

Let $W$ be a Borel subset of $I \times I$, where $I$ is the unit interval, such that for each $x$ in $I, W_{x}=\{y:(x, y) \in W\}$ is uncountable. The following parametrization problem arose from work of J. Choksi [2].

Is there a Borel isomorphism, $g$, of $I \times I$ onto $W$ such that for each $x$, $g(x, \cdot)$ maps $I$ onto $W_{x}$ ?

Choksi [2, p. 115] observed that a positive solution to this problem would greatly simplify some of his arguments. However, it is not necessarily true that $W$ has a Borel parametrization. In [6], the second author gives some necessary and sufficient conditions for $W$ to have a Borel parametrization. But not all is lost, since A. H. Stone [10] points out that in order to simplify Choksi's arguments it is only necessary that $g$ and $g^{-1}$ be universally (or absolutely) measurable.

We show in Theorem 6 of this paper that there is such a map $g$. In fact, both $g$ and $g^{-1}$ are measurable with respect to a very easily described family of universally measurable sets.

This particular family may be described as follows. Given a topological space $X, S(X)$ is the smallest family of subsets of $X$ containing the open sets and closed under complementation and operation (A) [5, p. 30]. It follows from the properties of operation (A), that $S(X)$ is also closed under countable

Received by the editors April 10, 1977 and, in revised form, July 20, 1977 and October 20, 1977.

AMS (MOS) subject classifications (1970). Primary 28A20; Secondary 04A15, 02K30, 54H05. 
unions [3, p. 107]. The study of this family was proposed by N. Lusin [5, p. 468]. O. Nikodým gave a method of constructing this family by alternating the operation (A) and the complementation operator and showed in uncountable Polish spaces that there are sets of arbitrarily high class. Kantorovitch and Livensohn showed that $S(X)$ is a subfamily of $\Delta_{2}^{1}(X)$, the family of all subsets of $X$ which are simultaneously PCA and CPCA sets [5, p. 468]. K. Kunugui [4] in a very penetrating study showed that $S(X)$ is a proper subfamily of $\Delta_{2}^{1}(X)$ provided $X$ is an uncountable Polish space. The sets in $S(X)$ are universally measurable; i.e., measurable with respect to the completion of every $\sigma$-finite measure defined on the Borel subsets of $X[5$, p. 95]. Of course, $S(X)$ is in general a proper subfamily of the family of all universally measurable sets.

Let us note that the existence of a parametrization, $g$, which is universally measurable has been shown by E. Wesley [14]. Wesley's arguments are of a meta-mathematical nature in that forcing techniques are employed. On the other hand, our arguments use only standard techniques of descriptive set theory.

Earlier, Wesley [12] proved, also by forcing methods, the following theorem and applied it to mathematical economics [13].

THEOREM. There is a function $h$ from $I \times I$ into $I$ such that

(1) $h$ is $\mathcal{L} \otimes \mathcal{L}$-measurable,

(2) for each $x, h(x, \cdot)$ is a Borel isomorphism of I onto $W_{x}$, and

(3) for each $y, h(\cdot, y)$ is an L-measurable selector of $W$.

That $h(\cdot, y)$ is a selector of $W$ means that for each $x, h(x, y) \in W_{x}$. Here $\mathcal{L}$ denotes the family of Lebesgue measurable subsets of $I$.

In this paper we strengthen this theorem of Wesley's as follows

THEOREM 7. Let $W$ be a Borel subset of $I \times I$ such that for each $x, W_{x}$ is uncountable. Then there is a map $h: I \times I$ into $I$ so that

(1) $h$ is an $S(I \times I)$ measurable map,

(2) for each $x, h(x, \cdot)$ is a Borel isomorphism of I onto $W_{x}$, and

(3) for each $y, h(\cdot, y)$ is an $S(I)$-measurable selector of $W$.

Let us note that (3) follows from (1) and (2). This implies that each $h(\cdot, y)$ is a universally measurable selector of $W$. Of course, Yankov [15] and von Neumann [7] have proven that $W$ has a selector which is $\mathscr{B}(\mathscr{Q}(I))$-measurable, where $\mathscr{B}(\mathscr{Q}(I))$ is the $\sigma$-algebra generated by the analytic subsets of $I$. Since $\mathscr{B}(\mathscr{Q}(I))$ is a proper subfamily of $S(I)$, the individual selector obtained by Yankov and von Neumann is "more describable" than the selectors $h(\cdot, y)$. However, our selectors are still universally measurable, their graphs are disjoint, and the graphs of the selectors $h(\cdot, y)$ fill up $W$ in a describable fashion. It may be that $h$ can be taken to be $\mathscr{B}(\mathscr{Q}(I \times I))$-measurable, but 
this problem is unsolved by us at this time. Let us mention that a fairly complete survey of measurable selection theorems has been given by $\mathrm{D}$. $\mathrm{H}$. Wagner [11].

Our first theorem contains the technical work needed for our results. It is the result of a careful study of the method used by von Neumann and Yankov. Some definitions are necessary.

Seq will denote the set of all finite sequences of positive integers regarded as a topological space with the discrete topology. If $s=\left(s_{1}, \ldots, s_{n}\right) \in$ Seq and $t=\left(t_{1}, \ldots, t_{m}\right) \in$ Seq, then $t$ is said to extend $s(t \supseteq s)$, if $m \geqslant n$, and $t_{i}=s_{i}$, for every $i, 1 \leqslant i \leqslant n$. Also, $s * t \equiv\left(s_{1}, \ldots, s_{n}, t_{1}, \ldots, t_{m}\right)$. $J$ will denote the space $N^{N}$, the space of all infinite sequences of positive integers with the product topology. Of course, $J$ is the zero-dimensional Baire space and may be regarded as the space of all irrational numbers between 0 and 1 via their continued fraction expansions. For any sequence $u=\left(u_{1}, u_{2}, \ldots\right)$ and any $n, u \mid n=\left(u_{1}, \ldots, u_{n}\right)$. If $s=\left(s_{1}, \ldots, s_{n}\right) \in \operatorname{Seq}$, then $J(s)$ is defined to be $\left\{\left(m_{1}, m_{2}, \ldots\right) \in N^{N}: m_{1}=s_{1}, \ldots, m_{n}=s_{n}\right\}$.

The symbol $Q_{2}$ will denote the space of all finite sequences of 0 's and 1's regarded as a topological space with the discrete topology. The letter $C$ will denote the Cantor set which we may regard as the space $\{0,1\}^{N}$ with the product topology. If $q=\left(q_{1}, \ldots, q_{n}\right) \in Q_{2}$, then $C(q)$ is defined to be $\left\{\left(t_{1}, t_{2}, \ldots\right) \in C: t_{1}=q_{1}, \ldots, t_{n}=q_{n}\right\}$.

THEOREM 1. Let $P$ be an analytic subset of $I \times J$ such that, for each $x, P_{x}$ is a nonempty perfect subset of $J$. Then there is a one-to-one map $F$ of $I \times C$ into $P$ so that

(1) $F$ is $\mathscr{B}(\mathscr{Q}(I \times C))$-measurable,

(2) $F^{-1}$ is $\mathscr{B}(\mathscr{Q}(I \times J))$-measurable, and

(3) for each $x$ in $I, F(x, \cdot)$ is a homeomorphism of $C$ into $\{x\} \times P_{x}$.

Proof. For each $x$ in $I$, let

Notice that since $P_{x}$ is closed,

$$
T_{x}=\left\{s \in \text { Seq: } P_{x} \cap J(s) \neq \varnothing\right\} .
$$

$$
P_{x}=\bigcap_{n=1}^{\infty}\left\{\sigma \in J: \sigma \mid n \in T_{x}\right\} .
$$

A map $f$ from $I \times Q_{2}$ into Seq will now be defined so that, for each $x$, $f(x, \cdot)$ maps $Q_{2}$ into $T_{x}$ in a one-to-one way. Let $\varnothing$ represent the empty sequence, both in $Q_{2}$ and Seq. The map $f$ is defined inductively. First, set $f(x, \varnothing)=\varnothing$. Given $f(x, r)=s_{r} \in T_{x}$, let $s$ be the shortest extension of $s_{r}$ such that for some positive integers, $m \neq n$, both $s * m$ and $s * n$ are in $T_{x}$. Let $m_{0}$ be the least such and $m_{1}$ the next. Then set $f(x, r * 0)=s * m_{0}$ and $f(x, r * 1)$ $=s * m_{1}$. 
Thus, $f$ maps $I \times Q_{2}$ into Seq. Let $G$ be the graph of $f(G=\{(x, q, t)$ : $f(x, q)=t\}$ ). We claim that $G$ is $\mathscr{B}(\mathscr{Q})$-measurable-that is (since $Q_{2}$ and Seq are countable sets with the discrete topology), for each $q$ and $t, G(q, t)=\{x$ : $f(x, q)=t\}$ is in $\mathscr{B}(\mathscr{Q}(I))$. This claim will be demonstrated later.

The function $F$ can now be defined. Let $f_{x}(t)=f(x, t)$. Notice that for each $x \in I$ and $\tau \in C, \cap_{n=1}^{\infty} J\left(f_{x}(\tau \mid n)\right)$ contains a single element of $P_{x}$. Define $F_{x}(\tau)$ to be this unique element of $\bigcap_{n=1}^{\infty} J\left(f_{x}(\tau \mid n)\right)$ and let $F(x, \tau)=$ $\left(x, F_{x}(\tau)\right)$.

It can be checked that $F$ is a one-to-one map of $I \times C$ into $P$.

Let us suspend the proof for a moment and make some remarks which may illuminate the preceding construction. For each $x, T_{x}$ is a subtree of Seq and, because $P_{x}$ is closed, $P_{x}$ can be regarded as the set of branches of $T_{x}$. The map $f_{x}$ at each stage picks the two left-most extensions which come from the previous stage in the definition of $f$. That there are at least two (incomparable) extensions of any node of $T_{x}$ follows from the fact that $P_{x}$ is dense in itself. It should also be noted that $T_{x}$ has no dead ends-that is, by the definition of $T_{x}$, every node has a branch from $P_{x}$ passing through it.

Let us verify that $F$ is $\mathscr{B}(\mathscr{Q}(I \times C))$-measurable. Let $V$ be an open subset of $I$. Then

$$
F^{-1}(V \times J(s))=(V \times C) \cap\{(x, \tau) \in I \times C: F(x, \tau) \in J(s)\} .
$$

Since $F_{x}(\tau)$ is the unique element of $\cap_{n=1}^{\infty} J\left(f_{x}(\tau \mid n)\right), F_{x}(\tau)$ is in $J(S)$ if and only if there is some $n$ so that $f_{x}(\tau \mid n)$ extends $s$. So,

$$
F^{-1}(V \times J(s))=(V \times C) \cap\left[\bigcup_{t \supseteq s} \bigcup_{n}\left\{(x, \tau): f_{x}(\tau \mid n)=t\right\}\right] .
$$

Now, each set in the union on the right-hand side is in $\mathscr{B}(\mathscr{Q}(I \times C))$. To be explicit:

$$
\begin{aligned}
\left\{(x, \tau): f_{x}(\tau \mid n)=t\right\} & =\bigcup_{q \in Q_{2}}\left\{(x, \tau): \tau \mid n=q \text { and } f_{x}(q)=t\right\} \\
& =\bigcup_{q \in Q_{2}}[\{(x, \tau): \tau \mid n=q\} \cap(G(q, t) \times C)] .
\end{aligned}
$$

Thus, in order to show that $F^{-1}(V \times J(s))$ is in $\mathscr{B}(\mathbb{Q}(I \times C))$, it suffices to show that $G(q, t)$ is in $\mathscr{B}(\mathscr{Q}(I))$. Recalling the inductive definition of the map $f$, we can construct the graph $G$ of $f$ in stages as follows:

$G_{0}=I \times\{\varnothing\} \times\{\varnothing\}$ and, for each $n$,

$G_{n+1}=\bigcup_{i=0,1} \cup_{m_{0}, m_{1} \in N} \cup_{q^{\prime} \in Q_{2}} \bigcup_{t^{\prime}, t^{\prime \prime} \in \operatorname{Seq}}\left\{(x, q, t):\left(x, q^{\prime}, t^{\prime}\right) \in G_{n}, t^{\prime \prime}\right.$ extends $t^{\prime}, t^{\prime \prime} * m_{0}$ and $t^{\prime \prime} * m_{1} \in T_{x}, m_{0}<m_{1}$ and $\left(\forall k \leqslant m_{1}\right)\left[t^{\prime \prime} * k \in T_{x} \rightarrow\right.$ ( $k=m_{0}$ or $\left.\left.k=m_{1}\right)\right]$ and $q=q^{\prime} * i, t=t^{\prime \prime} * m_{i}$ and if $s$ extends $t^{\prime}$ and there are integers $a$ and $b$ so that $s * a$ and $s * b \in T_{x}$, then $s$ extends $\left.t^{\prime \prime}\right\} . G_{0}$ is certainly in $\mathscr{B}(\mathscr{Q})$ and, since $T$ is analytic, $G_{n} \in \mathscr{B}(\mathscr{Q})$ implies $G_{n+1} \in \mathscr{B}(\mathbb{Q})$. It can be checked that the desired graph $G=\cup_{n} G_{n}$ and is therefore 
$\mathscr{B}(\mathbb{Q}(I))$-measurable. Thus, $F$ is $\mathscr{B}(\mathscr{Q}(I \times C))$-measurable.

To see that $F^{-1}$ is $\mathscr{B}(\mathbb{Q}(I \times J))$-measurable, notice that for $q \in Q_{2}$,

$$
T(q)=\left\{(x, y): y \in J\left(f_{x}(q)\right)\right\}=\bigcup_{s \in \operatorname{Seq}}[(I \times J(s)) \cap(G(q, s) \times J)] .
$$

It follows from this last equation that $T(q)$ is in $\mathscr{B}(\mathscr{Q}(I \times J))$.

Now, since

$$
\begin{aligned}
F(I \times C) & =\left\{(x, y): \exists \tau \in C \text { and }\{y\}=\bigcap_{n=1}^{\infty} J\left(f_{x}(\tau \mid n)\right)\right\} \\
& =\bigcup_{\tau \in C} \bigcap_{n=1}^{\infty}\left\{(x, y): y \in J\left(f_{x}(\tau \mid n)\right)\right\}
\end{aligned}
$$

we have

$$
F(I \times C)=\bigcap_{n=1}^{\infty}\left[\bigcup_{q \in\{0,1\}^{n}}\left\{(x, y): y \in J\left(f_{x}(q)\right)\right\}\right],
$$

and it follows that $F(I \times C)$ is in $\mathscr{B}(\mathbb{Q}(I \times J))$.

Next, for $q \in Q_{2}$ and $V$ open in $I$,

$$
\begin{aligned}
F(V \times C(q)) & =\left\{(x, y): x \in V \text { and } y \in J\left(f_{x}(q)\right)\right\} \cap F(I \times C), \\
& =(V \times J) \cap\left\{(x, y): y \in J\left(f_{x}(q)\right)\right\} \cap F(I \times C) .
\end{aligned}
$$

From this it follows that $F(V \times C(q))$ is in $\mathscr{B}(\mathscr{Q}(I \times J))$ and therefore $F^{-1}$ is $\mathscr{B}(\mathscr{Q}(I \times J))$-measurable.

Finally, for each $x$ in $I$,

$$
\begin{aligned}
F_{x}^{-1}(J(s)) & =\left\{\tau: \bigcap_{n=1}^{\infty} J\left(f_{x}(\tau \mid n)\right) \subseteq J(s)\right\} \\
& =\bigcup_{n=1}^{\infty}\left\{\tau: J\left(f_{x}(\tau \mid n)\right) \subseteq J(s)\right\} .
\end{aligned}
$$

So,

$$
F^{-1}(J(s))=\bigcup_{q \in K_{x}} C(q), \quad \text { where } K_{x}=\left\{q \in Q_{2}: f_{x}(q) \supseteq s\right\} .
$$

Thus, $F_{x}$ is a one-to-one continuous map of $C$ into $P_{x}$. Of course, this implies that $F_{x}$ is a homeomorphism.

This completes the proof of Theorem 1 .

The strategy for proving our main theorem calls for composing $\mathscr{B}(\mathbb{Q})$ measurable maps such as $F$. Since these compositions might not be $\mathscr{B}(\mathscr{Q})$ measurable, we consider the larger class of $S$-measurable functions, which are closed under compositions, as shown by the following lemma.

LEMMA 2. Let $g$ be a map of the topological space $X$ into the topological space 
$Y$. If $g^{-1}(U) \in S(X)$ for every open subset $U$ of $Y$, then $g^{-1}(E) \in S(X)$ for every $E \in S(Y)$.

Proof. Let $H=\left\{E: g^{-1}(E) \in S(X)\right\}$. It is easy to check that $H$ is closed under complementation and operation (A). Since $H$ contains the open subsets of $Y, S(Y) \subseteq H$.

Another observation is necessary.

Lemma 3. If $X$ and $Y$ are Polish spaces, $M$ is an analytic subset of $X \times Y$ and the subset $K$ of $X \times Y$ is defined by letting each $K_{x}$ be the dense-in-itself kernel of $M_{x}[3, p .136]$, then $K$ is analytic.

Proof. Let $T$ be the subset of $Y^{\omega}$ consisting of all sequences $\left(y_{n}\right)_{n=1}^{\infty}$ such that the set $\left\{y_{n}: n \in N\right\}$ is dense in itself. Then $T$ is a $G_{\delta}$ subset of $Y^{\omega}$. Let $Z=\left\{\left(x,\left(y_{n}\right)\right) \in X \times Y^{\omega}:\left(y_{n}\right) \in T\right.$ and $\left.(\forall n)\left(x, y_{n}\right) \in M\right\}$. Then $Z$ is an analytic subset of $X \times Y$. Since

$$
K=\left\{(x, y):(\exists p)\left(\exists\left(y_{n}\right)\right)\left[\left(x,\left(y_{n}\right)\right) \in Z \text { and } y=y_{p}\right]\right\},
$$

the set $K$ is analytic.

THEOREM 4. Let $M$ be an analytic subset of $I \times J$ such that $M_{x}$ is an uncountable closed set for every $x$. Then there is a one-to-one map $g$ of $I \times I$ onto $M$ such that

(1) $g$ and $g^{-1}$ are $S(I \times I)$-measurable and

(2) for each $x, g(x, \cdot)$ is a Borel isomorphism of $I$ onto $M_{x}$.

Proof. Let $P=\left\{(x, y): y\right.$ is a cluster point of $\left.M_{x}\right\}$. Then $P \subseteq M$ and, according to Lemma 3,P is an analytic subset of $I \times J$ such that each $P_{x}$ is nonempty and perfect.

Let $F$ be a map having the properties described in Theorem 1. Let $\theta$ be a Borel isomorphism of $I$ onto $C$ and let $k(x, y)=F(x, \theta(y))$, for each $(x, y) \in$ $I \times I$. Clearly, $k$ is an $S(I \times I)$-isomorphism of $I \times I$ onto $F(I \times C) \subseteq M$.

We will now give a Schröder-Bernstein type argument. Let $R=k(I \times I)$, $S_{0}=M-R$ and $T_{0}=(I \times I)-M$. Thus,

$$
\begin{aligned}
I \times I & =R \cup S_{0} \cup T_{0} \\
& =T_{0} \cup S_{0} \cup\left(T_{1} \cup S_{1}\right) \cup \cdots \cup\left(T_{n} \cup S_{n}\right) \cup \cdots \cup D,
\end{aligned}
$$

where $T_{n}=k^{n}\left(T_{0}\right), S_{n}=k^{n}\left(S_{0}\right)$ and $D=\bigcap_{p=1}^{\infty} k^{p}(R)$. Also,

$$
\begin{aligned}
M & =R \cup S_{0} \\
& =S_{0} \cup\left(T_{1} \cup S_{1}\right) \cup \cdots \cup\left(T_{n} \cup S_{n}\right) \cup \cdots \cup D \\
& =\left(T_{1} \cup S_{0}\right) \cup\left(T_{2} \cup S_{1}\right) \cup \cdots \cup\left(T_{n+1} \cup S_{n}\right) \cup \cdots \cup D .
\end{aligned}
$$

Set $H=D \cup \cup{ }_{n=0}^{\infty} S_{n}$ and $G=\cup_{n=0}^{\infty} T_{n}$ and define

$$
g(z)= \begin{cases}z, & \text { if } z \in H, \\ k(z), & \text { if } z \in G .\end{cases}
$$


It can be easily checked that $g$ is a one-to-one map of $I \times I$ onto $M$ and that, for each $x, g(x, \cdot)$ is a Borel isomorphism of $I$ onto $M_{x}$.

If $U$ is an open subset of $I \times I$, then

$$
g^{-1}(U)=g^{-1}(U \cap H) \cup g^{-1}(U \cap G)=(U \cap H) \cup k^{-1}(U \cap G) .
$$

It follows from Lemma 1 that the maps $k^{n}$ and $k^{-n}$ are $S\left(I^{2}\right)$-measurable for each $n$, so that the sets $S_{n}, T_{n}, H$ and $G$ are in the family $S\left(I^{2}\right)$. Thus, $g^{-1}(U)$ is in $S\left(I^{2}\right)$. Similarly, $\left(g^{-1}\right)^{-1}(U)=g(U)=g(U \cap H) \cup g(U \cap G)=(U$ $\cap H) \cup k(U \cap G)$, so $g^{-1}$ is $S\left(I^{2}\right)$-measurable.

Lemma 5. Let $W$ be a Borel subset of $I \times I$. Then there is a closed subset $M$ of $I \times J$ and a Borel isomorphism $\psi$ of $M$ onto $W$ such that, for each $x, \psi(x, \cdot)$ maps $M_{x}$ onto $W_{x}$.

Proof. There exist a closed subset $F$ of $J$ and $\phi=\left(\phi_{1}, \phi_{2}\right)$ a one-to-one continuous map of $F$ onto $W[5, \mathrm{pp} .441,447]$. Let $M=\left\{\left(\phi_{1}(y), y\right): y \in F\right\}$ and let $\psi=\phi \circ \pi_{2}$. Since $\pi_{2} \mid M$ is one-to-one, this is a Borel isomorphism and we are done.

The following theorem is a direct consequence of results (4) and (5).

THEOREM 6. Let $W$ be a Borel subset of $I \times I$ such that, for each $x$, $W_{x}=\{y:(x, y) \in W\}$ is uncountable. Then there is a one-to-one map $g$ of $I \times I$ onto $W$ such that:

(1) for each $x, g(x, \cdot)$ is a Borel isomorphism of I onto $W_{x}$ and

(2) both $g$ and $g^{-1}$ are $S(I \times I)$-measurable.

Our strengthened version of Wesley's theorem is now an easy corollary.

THEOREM 7. Let $W$ be a Borel subset of $I \times I$ such that, for each $x$ in $I, W_{x}$ is uncountable. Then there is a map $h$ from $I \times I$ into I such that:

(1) $h$ is $S(I \times I)$-measurable,

(2) for each $x, h(x, \cdot)$ is a Borel isomorphism of $I$ onto $W_{x}$, and

(3) for each $y, h(\cdot, y)$ is an $S(I)$-measurable selector of $W$.

Proof. Let the map $g$ be given by Theorem 6 and let $h=\pi_{2} \circ g$.

Let us remark that the proof of our theorem could have been greatly shortened if the following were true: if $W$ is a Borel subset of $I \times I$ such that each $W_{x}$ is uncountable, then $W$ contains a Borel set $B$ such that each $B_{x}$ is a nonempty perfect subset of $I$. However, this is not true, as the following example shows.

EXAMPLE. Let $C_{1}$ and $C_{2}$ be disjoint coanalytic subsets of $I$ which cannot be separated by Borel sets. Let $A_{i}=I-C_{i}, i=1,2$. Let $g_{i}$ be a continuous map of $J$ onto $A_{i}$ such that $g_{i}^{-1}(t)$ is uncountable for each $t \in A_{i}, i=1,2$. Let $W=W_{1} \cup W_{2}$, where

$$
W_{i}=\left\{\left(g_{i}(t), t\right): t \in J\right\} \quad \text { for } i=1,2 .
$$


Then $W$ is a Borel subset of $I \times I$ and each $W_{x}$ is uncountable. Now, if $W$ were to contain a Borel set $B$ such that each $B_{x}$ is nonempty and perfect (and therefore compact), then according to a theorem of Novikov [8], there would be a Borel set $\Gamma$ which uniformizes $B$. But, then $\pi_{1}\left(W_{2} \cap \Gamma\right)$ would be a Borel set separating $C_{1}$ from $C_{2}$.

On the other hand, the nontheorem discussed above has the following true approximation:

If $W$ is a Borel subset of $I \times I$ such that each $W_{x}$ is uncountable, then $W$ contains a Borel set $B$ such that, for all random $x, B_{x}$ is a nonempty perfect set. Here we use the word random in the sense of recursion theory-that is, a real $x$ is said to be random if the countable ordinal $\omega_{1}^{x}$ (the least ordinal not recursive in $x$ ) is equal to the least nonrecursive ordinal $\omega_{1}^{\text {Church-Kleene }}$. The set of random reals is Borel and was shown to have Lebesgue measure 1 by Sacks [9]; see [1] for further details. The proof of Theorem 1 can be modified for the above set $B$ to obtain a Borel map $F$ with the desired properties satisfied for all random $x$; then the techniques of Lemma 4 yield directly a Borel map $h$ for the set $W$ having the desired properties of Theorem 5 for all random $x$. For nonrandom $x$, the map $h$ can be filled in arbitrarily so that $h(x, \cdot)$ is a Borel isomorphism of $I$ onto $W_{x}$-the resulting map will still be Lebesgue measurable.

This shorter proof is in the spirit of Wesley's original argument, although the above can be done without reference to forcing or set theory, which are essential to Wesley's proof. Neither Wesley's approach nor the approach outlined above seem to give the precise describability of the map $h$ as $S$-measurable obtained in Theorem 5 .

Finally, let us note that Theorem 6 can be generalized to analytic sets. First we need an improvement of Theorem 1 . Since the argument is in many respects similar to Theorem 1, we shall outline the argument and not go into details.

THEOREM 8. Let $A$ be an analytic subset of $I \times J$ such that for each $x, A_{x}$ is uncountable. Then there is a one-to-one map $F$ of $I \times J$ into $A$ such that

(1) $F$ is $\mathscr{B}(\mathbb{Q}(I \times C))$-measurable,

(2) $F^{-1}$ is $\mathscr{B}(\mathbb{Q}(I \times J))$-measurable,

(3) for each $x, F(x, \cdot)$ is a homeomorphism of $C$ into $\{x\} \times A_{x}$.

Proof. Let $\{E(s): s \in$ Seq $\}$ be a Souslin scheme such that

$$
A=\bigcup_{\sigma \in J} \bigcap_{n=1}^{\infty} E(\sigma \mid n) .
$$

We also assume $E(s) \supseteq E(t)$, if $t$ extends $s$, the sets $E(s)$ are closed and of diameter $<1 /$ length $(s)+1$. For each $s \in$ Seq, set 


$$
A(s)=\bigcup_{t \in J(s)} \bigcap_{n=1} E(t \mid n) \subseteq E(s) .
$$

For each $x$ in $I$, let

$$
T_{x}=\left\{s \in \text { Seq: }(A(s))_{x} \text { is uncountable }\right\}
$$

and set

$$
T=\bigcup\left\{\{x\} \times T_{x}: x \in I\right\}
$$

Since

$$
T=\cup\{U(s) \times\{s\}: s \in \mathrm{Seq}\},
$$

where $U(s)=\left\{x:(A(s))_{x}\right.$ is uncountable $\}$, it follows that $T$ is an analytic subset of $I \times$ Seq.

A map $f$ from $I \times Q_{2}$ into Seq will now be inductively defined so that for each $x, f(x, \cdot)$ maps $Q_{2}$ into $T_{x}$. First, set $f(x, \varnothing)=\varnothing$. Given $f(x, r)=s_{r} \in$ $T_{x}$, let $\left(s_{0}, s_{1}\right)$ be the first pair in the lexicographical ordering of Seq $\times$ Seq so that $s_{0}$ and $s_{1}$ extend $s_{r}, s_{0}$ and $s_{1}$ are in $T_{x}$ and $\left(E\left(s_{0}\right) \cap E\left(s_{1}\right)\right)_{x}=\varnothing$. (At this point, we consider Seq to have the well order defined by $s \prec t$ if (1) length $(s)<$ length $(t)$ or (2) length $(s)=$ length $(t)$ and $s_{i}<t_{i}$, where $i$ the first coordinate in which $s$ and $t$ differ.) It can be checked that such a pair exists. Set $f(x, r * 0)=s_{0}$ and $f(x, r * 1)=s_{1}$.

It can be shown that the graph $G$ of $f$ in $I \times Q_{2} \times$ Seq is $B(Q)$ measurable.

Define $F_{x}(\tau)$ to be the unique element $\left(\bigcap_{n=1}^{\infty} E\left(f_{x}(\tau \mid n)\right)\right)_{x}$ for each $(x, \tau)$ $\in I \times C$ and let $F(x, \tau)=\left(x, F_{x}(\tau)\right)$.

The proof that the map $F$ meets the three requirements of the theorem is similar to that given in Theorem 1 .

Using Theorem 8 together with a Schröder-Bernstein type argument we obtain the following theorem.

THEOREM 9. Let $A$ be an analytic subset of $I \times I$ such that for each $x, A_{x}$ is uncountable. Then there is a one-to-one map $g$ of $I \times I$ onto A such that

(1) for each $x, g(x, \cdot)$ is a $\mathfrak{B}(\mathbb{Q}(I))$-measurable isomorphism of I onto $A_{x}$.

(2) both $g$ and $g^{-1}$ are $S(I \times I)$-measurable.

\section{REFERENCES}

1. D. Cenzer and R. D. Mauldin, Inductive definability, measure and category (to appear).

2. J. R. Choksi, Measurable transformations on compact groups, Trans. Amer. Math. Soc. 184 (1973), 101-124.

3. F. Hausdorff, Set theory, Chelsea, New York, 1964.

4. K. Kunugui, Sur un théorème d'existence dans la théorie des ensembles projectifs, Fund. Math. 29 (1937), 169-181.

5. K. Kuratowski, Topology, Vol. I, Academic Press, New York, 1966.

6. R. D. Mauldin, Borel parameterizations (preprint).

7. J. von Neumann, On rings of operators; reduction theory, Ann. of Math. 30 (1949), 401-485. 
8. P. S. Novikov, Sur les projections de certains ensembles mesurables B, Dokl. Akad. Nauk. SSSR (N.S.) 23 (1939), 864-865.

9. G. E. Sacks, Measure-theoretic uniformity, Trans. Amer. Math. Soc. 142 (1969), 381-420.

10. A. H. Stone, Measure theory, Lecture Notes in Math., vol. 541, Springer-Verlag, Berlin and New York, 1976, pp. 43-48.

11. D. H. Wagner, Survey of measurable selection theorems, SIAM J. Control Optimization 15 (1977), 859-903.

12. E. Wesley, Extensions of the measurable choice theorem by means of forcing, Israel J. Math. 14 (1973), $104-114$.

13. , Borel preference orders in markets with a continum of traders, J. Math. Econom. 3 (1976), 155-165.

14.

15. W. Yankov, Sur l'uniformisation des ensembles A, Dokl. Akad. Nauk SSSR (N.S.) 30 (1941), 597-598.

Department of Mathematics, University of Florida, Gaingsville, Florida 32611

Department of Mathematics, North Texas State University, Denton, Texas 76203 